\title{
The role of polyclonal intravenous immunoglobulin in treating HIV-infected children with severe bacterial infections: $A$ retrospective cohort study
}

\author{
Lyen C Huang1, Landon Myer ${ }^{1,2}$ and Heather B Jaspan*3,4
}

\begin{abstract}
Address: ${ }^{1}$ School of Public Health and Family Medicine, University of Cape Town, Faculty of Health Sciences, Anzio Road, Observatory 7925 , Cape Town, South Africa, ${ }^{2}$ Department of Epidemiology, Mailman School of Public Health, Columbia University, 722 West 168 th Street, New York, NY, USA, ${ }^{3}$ Desmond Tutu HIV Centre, Institute of Infectious Disease and Molecular Medicine, University of Cape Town, Anzio Road, Observatory 7925, Cape Town, South Africa and ${ }^{4}$ Children's Hospital and Regional Medical Centre, University of Washington, 4800 Sandpoint Way NE, Seattle, WA 98105, USA

Email: Lyen C Huang - lyen.huang@gmail.com; Landon Myer - landon.myer@uct.ac.za; Heather B Jaspan* - hbjaspan@hotmail.com

* Corresponding author
\end{abstract}

Published: 24 September 2008

BMC Infectious Diseases 2008, 8:127 doi:10.1186/147|-2334-8-127
Received: 7 March 2008

Accepted: 24 September 2008

This article is available from: http://www.biomedcentral.com/I47/-2334/8/I27

(c) 2008 Huang et al; licensee BioMed Central Ltd.

This is an Open Access article distributed under the terms of the Creative Commons Attribution License (http://creativecommons.org/licenses/by/2.0), which permits unrestricted use, distribution, and reproduction in any medium, provided the original work is properly cited.

\begin{abstract}
Background: Mortality among HIV-infected children in developing countries remains high after serious bacterial infections despite the use of antibiotics. Intravenous immunoglobulin (IVIG) has been used as an adjuvant therapy to treat these infections, but little data exists regarding its efficacy, and previous studies have focused on IVIG as a prophylactic agent. We examined the impact of IVIG as an adjuvant therapy in reducing mortality and length of hospital stay in HIV-infected children with serious bacterial infections.
\end{abstract}

Methods: This retrospective study focused on pediatric admissions at a large urban hospital between 2002 and 2006. Children between the ages of one month and nine years of age with laboratory confirmed HIV-status, serious bacterial infection, no prior exposure to IVIG, and a hospital length of stay of 5 days or more, were eligible for inclusion.

Results: A total of 140 children (median age 1.2 years) met inclusion criteria; lower respiratory tract infection was diagnosed in $94(67 \%)$ of the children, while $74(53 \%)$ had bacterial sepsis. Fiftyfour $(39 \%)$ children were receiving antiretroviral therapy and 39 (28\%) were receiving tuberculosis treatment. Overall $73(52 \%)$ were treated with IVIG, with the majority $(74 \%)$ of children receiving a single dose. Thirteen (9\%) died during their hospital admission. In crude analysis IVIG was significantly associated with increased mortality was (Odds Ratio (OR): 5.8; 95\% Confidence Interval (Cl): I.2-27.I) and this association was weakened by adjustment for other predictors of mortality (OR 4.3, 95\% Cl 0.7-27.9, $\mathrm{p}=0.123)$. IVIG use was also associated with longer hospital stays.

Conclusion: Administration of one to three doses of IVIG during the acute phase of illness does not appear to reduce mortality or the length of hospital stays in HIV-infected children with serious bacterial infections. However, the retrospective nature of this study makes confounding by indication difficult to control and further studies regarding the timing, dosing, and method of administration are required. Nonetheless the routine use of IVIG in resource-limited settings should be carefully considered given its high cost. 


\section{Background}

With 2.3 million children living with HIV worldwide in 2006 [1], the virus is a particular health threat to children in underdeveloped settings. South Africa has been heavily affected by the epidemic: in 2005, 30\% of pregnant women in South Africa were HIV-infected and an estimated 240,000 children were infected from mother to child transmission. This number is expected to increase in the coming years [2], placing additional strain on an already over-burdened health system.

Infectious diseases are the leading cause of death among HIV-infected children in South Africa and many other developing countries, where as much as two-thirds of all under-5 deaths may be directly or indirectly related to HIV [3]. HIV-infected children are at significant risk of developing bacteremia and pneumonia, which are the most common causes of mortality among the children requiring hospital admission in South Africa [4-6].

The high mortality from infectious diseases in HIVinfected children despite intravenous (IV) antibiotic therapy has led to a search for adjuvant therapies such as intravenous immunoglobulin (IVIG). IVIG is an important component in treating some immunodeficiencies as well as autoimmune diseases [7]. For these diseases, immunoglobulin supplementation has several beneficial effects including primary antibody replacement, immune system modulation, suppression of auto-antibodies, and targeting of bacterial super-antigens [8,9]. Unfortunately, the theoretical benefits of IVIG administration to HIVinfected individuals have not been well-supported in the clinical setting. Two randomized controlled trials were unable to demonstrate any reduction in mortality from the prophylactic use of IVIG against serious bacterial infections, although some reduction in the number of hospitalizations and infections were seen $[10,11]$.

The role of IVIG in therapeutic treatment of acute infections in other pediatric populations appears somewhat promising, although no studies have been done specifically in HIV-infected children. One prospective study found that therapeutic IVIG was strongly associated with shorter stays in a pediatric ward as well as decreased complications and mortality regardless of HIV status [12]. Meta-analyses have demonstrated some therapeutic benefit in treating sepsis in HIV negative neonates, but not other age groups $[13,14]$. In the adult HIV-negative population, there is conflicting evidence regarding the effect of therapeutic benefits of IVIG on overall mortality $[15,16]$.

Despite the mixed nature of available data, IVIG continues to be used in conjunction with antibiotics to treat bacterial infections in some settings, particularly in HIVinfected children. However, the high cost of IVIG coupled with supply shortages due to increasing off-label use have resulted in calls for better guidelines and further research $[7,17,18]$.

To our knowledge, no study has evaluated IVIG as adjunct treatment for serious bacterial infections in HIV-infected children, although its use for sepsis prevention has been evaluated. We analyzed the impact of therapeutic IVIG administration on clinical outcomes among HIV-infected children hospitalized for serious bacterial infections in Cape Town, South Africa.

\section{Methods}

\section{Study design and population}

We conducted a retrospective cohort study of all HIVinfected pediatric admissions at an urban public sector hospital in Cape Town between January 2002 and June 2006. Over $50 \%$ of all admissions to the ward are HIVinfected; the most common presenting conditions were acute gastroenteritis or lower respiratory tract infections. The referral population is a largely black African population living in rapidly growing settlements with residents of predominantly low socioeconomic status.

All children between one month and nine years of age with laboratory confirmed HIV-status, a diagnosis of bacterial sepsis, bacterial pneumonia, and/or meningitis, no prior exposure to IVIG, and a hospital length of stay of 5 days or more, were eligible for inclusion. Exclusion criteria included established indications for IVIG such as Kawasaki's disease, autoimmune hemolytic anemia, Guillain-Barré syndrome, or idiopathic thrombocytopenia.

Data were collected from paper charts and cross-referenced with pharmacy supply logs to confirm IVIG dosing. For IVIG unexposed patients, the first admission for each patient meeting inclusion criteria after January 1, 2002, was chosen for analysis. For IVIG exposed patients, the first admission where the child received IVIG was used. Subsequent admissions were not examined.

Approval for this study was obtained from the University of Cape Town Faculty of Health Sciences Research Ethics Committee.

\section{Measures}

Basic demographic, laboratory and clinical information were abstracted from patient charts using a standardized abstraction form. Data were collected by a single individual. Data validity was checked by repeated blinded abstraction on randomly selected charts.

Electrolyte, metabolic, liver function, and hematological laboratory data was collected at four time points: admission, IVIG administration (or one week after admission 
for the unexposed group), one week after IVIG administration (or two weeks after admission for the unexposed group), and discharge. If data was not available on the exact date specified, lab values from the nearest date were used. CD4/8 counts and percentages were not recorded as part of this study due to the unavailability of these measures for most participants near the time of diagnosis.

Diagnoses of bacterial sepsis and bacterial pneumonia were included based on clinical findings including chest radiograph and blood cultures when available. A diagnosis of meningitis was included based on results of cerebrospinal fluid cultures collected from lumbar punctures. Protein-energy malnutrition (PEM) was used to describe clinical diagnoses of marasmus, Kwashiorkor or marasmic Kwashiorkor.

Hospital length of stay was defined as a binary outcome with a "long" stay defined as greater than the median length of stay for all patients. Mortality was considered only in the context of hospitalization. Patients who died after discharge were excluded from mortality totals since the date and cause of death could not be accurately discerned.

Antibiotics were analyzed individually and also grouped by relative breadth of organism coverage. Thus, first-line antibiotics were generally used as initial empiric coverage for sensitive organisms whereas fourth-line antibiotics were reserved for very resistant organisms or patients otherwise refractory to treatment. First-line antibiotics were defined as penicillin, ampicillin, gentamycin, ceftriaxone, and cefuroxime. Second-line antibiotics were cloxacillin, intravenous augmentin, cefazolin, and cefotaxime. Thirdline antibiotics were piperacillin, piperacillin plus tazobactam, amikacin, cefipime, and ceftazadime. Fourth-line antibiotics were ertapenem, imipenem, meropenem, and vancomycin.

A modified PRISM III score was used to grade the severity of illness in eligible children. The PRISM III is a validated physiologically-based score for evaluating the risk of mortality [19]. Because not all of the data required for calculating a complete PRISM III score were available upon chart review, we developed a modified scoring system based on percentages. A PRISM III score was calculated from the available data for each patient and then divided by the maximum possible score that the patient could have received. When more than one lab value was applicable, the most severe lab values were used to reflect the greatest morbidity during the course of illness.

\section{Analysis}

Data analysis was conducted using Stata 9.0 (Stata Corporation, College Station, Texas, United States). Data were described using means, medians and proportions; bivariate analyses employed Students' t-tests, Wilcoxon ranksum tests, and chi-square tests, as appropriate. Multiple logistic regression models were used to identify independent predictors of IVIG exposure, mortality, and hospital length of stay. Models were constructed by sequential addition of predictors and comparison of models using the Akaike information criteria and the likelihood-ratio chi-squared value. Variables were eligible to be examined in the model if missing data on a particular covariate did not reduce the number of observations in the model by more than $10 \%$ of the total cohort size.

\section{Results \\ Demographics}

Of 247 patient records reviewed, 140 children met the inclusion criteria for the cohort. The main reasons for exclusion were lack of a qualifying diagnosis, unconfirmed HIV status, hospital length of stay less than 5 days, use of IVIG for Kawasaki's disease and hemolytic anemia. The demographic characteristics of the 140 included patients are presented in Table 1.

In the cohort, 64 children (46\%) were female, and the median age was 1.2 years old (Interquartile Range (IQR): $0.5-2.3)$. The median weight on admission was $6.74 \mathrm{~kg}$ (IQR 4.71-9.56). Only 66 subjects had birth weights recorded, with a median weight of $2.79 \mathrm{~kg}$ (IQR 2.77 3.02). A subset of children were on highly active antiretroviral therapy (HAART) and tuberculosis (TB) treatment (39\% and $28 \%$ respectively).

\section{Hospital course}

The median length of stay was 14.5 days (IQR 7-35). Eighty-five children (61\%) had at least one positive blood culture during the hospital admission. Antibiotic use was common, with $118(84 \%)$ receiving $1^{\text {st-line antibiotics, }}$ $42(30 \%)$ receiving $2^{\text {nd }}-$ line antibiotics, $51(36 \%)$ receiving $3^{\text {rd }}-$ line antibiotics, and $44(31 \%)$ receiving $4^{\text {th-line }}$ antibiotics.

A diagnosis of lower respiratory tract infection was made in $94(67 \%)$ children, while $74(53 \%)$ were diagnosed with bacterial sepsis (proven or presumed). Only 4 (3\%) children were found to have bacterial meningitis. More than one qualifying diagnosis for inclusion was found in $31(22 \%)$ of the children.

Overall, 73 (52\%) children were exposed to IVIG. Dosing was always $400-500 \mathrm{mg} / \mathrm{kg}$ of IVIG given over 3-4 hours of infusion. A single dose of IVIG was administered to 54 (74\%) children, whereas $15(21 \%)$ received two doses and $4(5 \%)$ received three doses. During the total observation time, $13(9 \%)$ children died in hospital with the remainder being discharged. 
Table I: Demographic description of I 40 admissions, overall and stratified by IVIG exposure

\begin{tabular}{|c|c|c|c|c|}
\hline Characteristic & $\begin{array}{l}\text { Total cohort } \\
\mathrm{n}(\%)\end{array}$ & $\begin{array}{l}\text { IVIG Unexposed } \\
n \text { (\% of unexposed) }\end{array}$ & $\begin{array}{l}\text { IVIG Exposed } \\
n \text { (\% of exposed) }\end{array}$ & $\mathrm{P}$-value \\
\hline IVIG exposure (\% of total cohort) & & $68(48)$ & $73(52)$ & \\
\hline \multicolumn{5}{|l|}{ Gender } \\
\hline Males & $76(54)$ & $37(55)$ & $39(53)$ & 0.831 \\
\hline Females & $65(46)$ & $30(45)$ & $34(47)$ & \\
\hline On HAART & $54(39)$ & $2 I(3 I)$ & $33(45)$ & 0.092 \\
\hline On TB medication & $39(28)$ & $17(25)$ & $22(30)$ & 0.496 \\
\hline Mortality & $13(9)$ & $2(3)$ & $11(15)$ & 0.014 \\
\hline "Long" hospital stays & $65(46)$ & $12(18)$ & $53(73)$ & $<0.001$ \\
\hline \multicolumn{5}{|l|}{ Age at admission (yrs) } \\
\hline Median (IQR) & I.2(0.5-2.3) & $1.3(0.6-2.5)$ & $1.0(0.4-2.1)$ & 0.120 \\
\hline \multicolumn{5}{|l|}{ Weight at admission (kg) } \\
\hline Median (IQR) & $6.7(4.7-9.5)$ & $7.3(5.0-10.9)$ & $6.0(4.6-8.3)$ & 0.039 \\
\hline \multicolumn{5}{|l|}{ Temp. at admission $\left({ }^{\circ} \mathrm{C}\right)$} \\
\hline Median (IQR) & $37.3(36.5-38.2)$ & $37.4(36.5-38.2)$ & $37.3(36.5-38.5)$ & 0.812 \\
\hline \multicolumn{5}{|l|}{ Birth weight (kg) } \\
\hline Mean $(95 \% \mathrm{Cl})$ & $2.9(2.8-3.0)$ & $3.0(2.8-3.2)$ & $2.8(2.7-3.0)$ & 0.321 \\
\hline \multicolumn{5}{|l|}{ Hospital length of stay (days) } \\
\hline Median (IQR) & $15(7-35)$ & $8(6-12)$ & $30(15-59)$ & $<0.001$ \\
\hline \multicolumn{5}{|l|}{ Modified PRISM percentage } \\
\hline Median (IQR) & ||$(0-2 \mid)$ & $6(0-15)$ & $14(6-28)$ & 0.004 \\
\hline \multicolumn{5}{|l|}{ Hemoglobin on admission } \\
\hline Median (IQR) & $9.1(8.1-10.3)$ & $9.3(8.4-10.5)$ & $8.7(8.0-10.2)$ & 0.202 \\
\hline \multicolumn{5}{|l|}{ Diagnosis } \\
\hline Lower respiratory tract infection & $94(67)$ & $50(75)$ & $44(60)$ & 0.062 \\
\hline Bacterial sepsis & $74(53)$ & $24(36)$ & $50(68)$ & $<0.001$ \\
\hline Bacterial meningitis & $4(3)$ & $2(3)$ & $2(3)$ & 0.931 \\
\hline $\mathrm{TB}$ & $40(29)$ & $16(24)$ & $24(33)$ & 0.239 \\
\hline TB meningitis & $2(1)$ & $I(2)$ & $I(I)$ & 0.951 \\
\hline Gastroenteritis & $54(39)$ & $2 \mid(3 \mid)$ & $33(45)$ & 0.092 \\
\hline Urinary tract infection & $15(11)$ & $4(6)$ & $11(15)$ & 0.082 \\
\hline PEM & $16(11)$ & $7(10)$ & $9(12)$ & 0.727 \\
\hline Fungal sepsis & $4(3)$ & $0(0)$ & $4(5)$ & 0.052 \\
\hline Failure to thrive & $29(21)$ & $8(12)$ & $21(29)$ & 0.014 \\
\hline \multicolumn{5}{|l|}{ Cultures } \\
\hline Any positive blood culture & $85(61)$ & $29(43)$ & $56(77)$ & $<0.001$ \\
\hline Positive E. coli blood culture & $4(3)$ & $2(3)$ & $2(3)$ & 0.931 \\
\hline Positive S. pneumoniae blood culture & $21(15)$ & $10(15)$ & $11(15)$ & 0.981 \\
\hline Positive $C$. albicans urine culture & $7(5)$ & $0(0)$ & $7(10)$ & 0.009 \\
\hline \multicolumn{5}{|l|}{ Received } \\
\hline Ist-line antibiotics & II 8 (84) & $53(79)$ & $65(89)$ & 0.107 \\
\hline 2nd-line antibiotics & $42(30)$ & $12(18)$ & $30(4 I)$ & 0.003 \\
\hline 3rd-line antibiotics & $51(36)$ & $10(15)$ & $41(56)$ & $<0.001$ \\
\hline 4th-line antibiotics & $44(31)$ & $5(7)$ & $39(53)$ & $<0.001$ \\
\hline IV ampicillin & $92(66)$ & $37(55)$ & $55(75)$ & 0.012 \\
\hline IV cefotaxime & $32(23)$ & $6(9)$ & $26(36)$ & $<0.001$ \\
\hline IV vancomycin & $21(15)$ & $2(3)$ & $19(26)$ & $<0.001$ \\
\hline IV piperacillin/tazobactam & $21(15)$ & $4(6)$ & $17(23)$ & 0.004 \\
\hline IV fluconazole & $14(10)$ & $0(0)$ & $14(19)$ & $<0.001$ \\
\hline IV amphotericin & $8(6)$ & $0(0)$ & $8(\mathrm{II})^{\prime}$ & 0.005 \\
\hline
\end{tabular}

\section{Predicting IVIG exposure}

Bivariate analysis revealed significant associations between IVIG and mortality ( $\mathrm{p}=0.014)$, "long" hospital stays $(\mathrm{p}<0.001)$, presumed or proven bacterial sepsis $(\mathrm{p}$ $<0.0001)$, a diagnosis of failure to thrive $(\mathrm{p}=0.014)$, at least one positive blood culture $(\mathrm{p}<0.0001)$, and exposure to $2^{\text {nd }}-(\mathrm{p}=0.003), 3^{\text {rd }}-(\mathrm{p}<0.0001)$, and $4^{\text {th }}$-line antibiotics $(\mathrm{p}<0.0001)$. Specific antibiotics were also associated with IVIG, such as intravenous ampicillin $(\mathrm{p}=$ $0.012)$, cefotaxime $(\mathrm{p}<0.001)$, vancomycin $(\mathrm{p}<0.001)$, 
and piperacillin/tazobactam $(\mathrm{p}=0.004)$. Severity of illness, as measured by the modified PRISM score, was also associated with IVIG exposure $(p=0.004)$.

The best-fit logistic regression predicting IVIG exposure is presented in Table 2. Significant predictors were any positive blood culture regardless of organism (OR 4.3, 95\% CI 1.6-11.8), a diagnosis of failure to thrive (OR 5.0, 95\% CI 1.5-16.3), exposure to third-line (OR 4.0, 95\% CI 1.411.5) and fourth-line antibiotics (OR 10.3, 95\% CI 2.937.0), as well as intravenous ampicillin (OR 5.1, 95\% CI 1.7-15.7) and cefotaxime (OR 6.2, 95\% CI 1.7-22.5). The modified PRISM percentage was not a significant predictor of IVIG exposure in the multivariate analysis. Not included in the model are several variables in the analysis which were perfect predictors of IVIG exposure namely: diagnosis of fungal culture-proven sepsis, exposure to intravenous fluconazole, intravenous amphotericin, and positive urine culture for Candida albicans.

\section{Predicting mortality and hospital length of stay}

The best-fit logistic regression models for predicting mortality and hospital length of stays are presented in Table 3. The unadjusted odds ratio of IVIG association with mortality was 5.8 (95\% CI 1.2-27.1). However, the association became statistically non-significant after inclusion of significant predictors of mortality (OR 4.3, 95\% CI $0.7-$ 27.9). Significant predictors for mortality included exposure to intravenous vancomycin (OR 7.2, 95\% CI 1.533.4), a diagnosis of PEM (OR 13.3, 95\% CI 2.2-81.0), and a positive E. coli blood culture (OR 20.7, 95\% CI 1.1379.7). Being female was also significant for predicting mortality (OR 7.0, 95\% CI 1.3-38.7).

"Long" hospital stays were positively predicted by IVIG exposure (OR 7.5, 95\% CI 2.8-20.0) even after adjust-

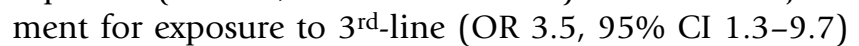

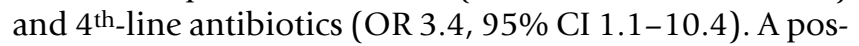
itive blood culture for Streptococcus pneumoniae (OR 0.13, 95\% CI 0.03-0.6) was protective against a "long" hospital stay.

\section{Discussion}

Our analysis suggests that one to three doses of IVIG provides only limited therapeutic benefit in acutely treating

Table 2: Logistic regression model predicting IVIG exposure

\begin{tabular}{ll}
\hline Predictors & Adjusted IVIG exposure OR $(95 \% \mathrm{Cl})$ \\
\hline Any positive blood culture & $4.3(1.6-11.8)$ \\
Diagnosis of failure to thrive & $5.0(1.5-16.3)$ \\
3rd-line antibiotics & $4.0(1.4-11.5)$ \\
4th-line antibiotics & $10.3(2.9-37.0)$ \\
IV ampicillin & $5.1(1.7-15.7)$ \\
IV cefotaxime & $6.2(1.7-22.5)$ \\
\end{tabular}

severe bacterial infections for HIV-infected children. Patients who received IVIG had longer hospital stays and a trend toward increased likelihood of mortality than those who did not, even after controlling for severity of illness and concomitant antibiotic exposure. Although a small benefit cannot be ruled out, the likelihood of a clinically significant benefit from IVIG appears to be low.

Theoretically, IVIG might have provided similar benefits in HIV-infection as seen in primary immunodeficiencies. However, differences in disease mechanism may explain why clinical outcomes have not been as significantly favorable in HIV-infection. HIV-infection in children often results in a relative hypergammaglobulinemia in comparison with adults, likely secondary to abnormal B-cell activation [20,21]. Despite the high immunoglobulin G (IgG) levels in children with HIV, measurement of these levels does not appear to be a good marker for humoral immune system functionality as these children often have a decreased response to vaccines [22-24]. This suggests that the poor antibody response is functional rather than quantitative in nature and simply elevating circulating IgG levels is insufficient to convey improved bacterial resistance in children.

Differences in study populations make comparison with other studies difficult. El-Nawawy et al. demonstrated that mortality in patients who received IVIG was halved from 56 to 28 percent [12]. Meta-analyses have shown very limited benefit of treating neonates, and even then only in lab-proven infection $[13,16]$. None of these studies distinguished between HIV-infected and non-infected children.

Thus far, the studies conducted on HIV-infected children have only examined the role of IVIG as prophylaxis. The NICHD trial showed increased time free of infections and decreases in serious bacterial infections and acute hospitalizations [10]. The PACTG protocol 51 showed that IVIG and zidovudine together reduced mild bacterial infections and acute hospitalizations [11]. However, the benefits of IVIG disappeared in children who also received daily cotrimoxazole. In both studies, mortality remained unchanged.

Differences in the timing and dosing of IVIG also make comparison across studies difficult. In this study, the majority of participants received only a single dose of $400-500 \mathrm{mg} / \mathrm{kg}$ IVIG. This dose was given when patients were significantly ill. Only two of the 21 trials included in the meta-analysis by Pildal and Getzsche administered IVIG in a single dose [15]. The other 19 trials administered treatment at intervals or continuously over the course of 1.5 to 6 days. Those in the study by El-Nawawy, et al., received equal doses administered during the first 3 days of admission [12]. Single doses of IVIG may be insuffi- 
Table 3: Logistic regression models predicting mortality and hospital length of stay

\begin{tabular}{lllll}
\hline Predictors & $\begin{array}{l}\text { Unadjusted Mortality OR } \\
(95 \% \mathrm{Cl})\end{array}$ & $\begin{array}{l}\text { Adjusted Mortality OR } \\
(95 \% \mathrm{Cl})\end{array}$ & $\begin{array}{l}\text { Unadjusted "Long" hospital } \\
\text { stays OR (95\% Cl) }\end{array}$ & $\begin{array}{l}\text { Adjusted "Long" hospital } \\
\text { stays OR (95\% Cl) }\end{array}$ \\
\hline IVIG exposure & $5.8(1.2-27.1)$ & $4.3(0.7-27.9)$ & $12.1(5.4-27.3)$ & $7.5(2.8-20.0)$ \\
\hline IV vancomycin & & $7.2(1.5-33.4)$ & \\
Diagnosis of PEM & $13.3(2.2-81.0)$ & $3.5(1.3-9.7)$ \\
E. coli blood culture & $20.7(1.1-379.7)$ & $3.4(1.1-10.4)$ \\
Female & $7.0(1.3-38.7)$ & $0.13(0.03-0.6)$ \\
3rd-line antibiotics & & & \\
4th-line antibiotics & & & \\
Positive blood culture for & & & \\
S. pneumoniae & & & \\
\hline
\end{tabular}

cient to provide protective effects, as it can take 4-6 months of infusion to reach a steady state [25]. The need to reach adequate protective serum levels is supported by the delay in treatment effect noted during the NICHD trial [26].

There may be several reasons why IVIG as well as vancomycin were associated with increased mortality. Late recognition of the severity of illness and delayed administration of IVIG could explain why treated children had longer hospital stays. In turn, long hospital stays lead to an increased risk of nosocomial infection potentially resistant to antibiotics and unaffected by IVIG.

Alternatively, many infections may have been viral or mycobacterial, thus explaining the ineffectiveness of antibiotics. Of the 13 children who died in-hospital, 4 had negative blood cultures for their entire stay. Excessive use of antibiotics may also have contributed to the increased mortality. The current practice in some settings, including this one, is that children receive a full course of antibiotics even if blood cultures are negative. This is due to concerns about the poor sensitivity of cultures. The overuse of antibiotics in turn may lead to the development of resistant organisms. A recent study in Tanzania found almost 14\% of pediatric admissions had laboratory-confirmed bacterial sepsis despite two-thirds of them having received prior antibiotic treatment [27]. Prolonged exposure to broad spectrum antibiotics also increases the risk of developing fungal infections which are not easily recognized or treated.

Mortality in the study cohort was also associated with PEM. This is consistent with previous studies showing the combination of malnutrition and HIV increases the likelihood of mortality $[28,29]$. This suggests that preventative care and ensuring adequate nutrition may help mitigate the severity of illness and reduce mortality. However, reducing mortality from malnutrition in the context of
HIV has been challenging and the interplay between various contributing factors remains unclear [30,31].

There were several limitations to this study. Retrospective studies cannot control which participants receive treatment, resulting in confounding by indication. Children who were more seriously ill and at greater risk of mortality were also those most likely to receive IVIG. Although an attempt to capture illness severity was done through a modified PRISM percentage, it may not have been an adequate indicator of severity. Nor has this modified score been validated in other studies. In addition, insufficient data on CD4 percentages and viral loads was available to take into account the level of immunosuppression. In the NICHD trial, only children with CD 4 counts $\geq 200 / \mathrm{mm}^{3}$ benefited from IVIG prophylaxis [10]. Conducting a prospective trial would address these limitations and allow for better subgroup analysis. Also, a future comparison between intravenous and subcutaneous administration may be warranted. Subcutaneous administration has become more commonplace due to its improved side effect profile, equivalent benefit, and improved quality of life, and in some cases lower costs [32]. Whether these benefits would be seen in resource-limited settings remains to be seen.

\section{Conclusion}

To our knowledge, this is the first study to evaluate IVIG as adjunct treatment for bacterial sepsis in HIV-infected children. Previous studies have focused on sepsis prevention. Our findings suggest that one to three doses of IVIG during the acute phase of illness may have minimal to no therapeutic benefit in reducing mortality and is not associated with decreased length of hospital stay in HIVinfected children with serious bacterial infections. However, the retrospective nature of this study makes confounding by indication difficult to control. Further research will be necessary regarding whether different timing, dosing, and/or methods of administration may prove 
more beneficial. The benefits seen by other studies suggest earlier and/or longer administration of immunoglobulin may be more effective. However, in limited-resource settings, non-pharmacological improvements in disease prevention, detection, and follow-up care should also be strongly considered in reducing the significant morbidity and mortality seen in young infants and children living with HIV.

\section{Competing interests}

The authors declare that they have no competing interests.

\section{Authors' contributions}

LCH conducted the background literature review, data collection, statistical analysis, and writing of the paper. LM supervised the study design, statistical analysis, and revised the manuscript. HBJ conceived of the study, provided clinical expertise, and revised the manuscript. All authors read and approved the final manuscript.

\section{Acknowledgements}

The authors would like to thank Rebecca Kim for her biostatistical advice and help in editing the manuscript.

\section{References}

I. UNAIDS/WHO: UNAIDS/WHO AIDS Epidemic Update: December 2006. 2006 [http://www.unaids.org/en/KnowledgeCen tre/HIVData/EpiUpdate/EpiUpdArchive/2006/Default.asp]. Geneva, Switzerland: UNAIDS/WHO

2. South African Department of Health: National HIV and Syphilis Antenatal Sero-prevalence Survey in South Africa 2005. 2005 [http://www.doh.gov.za/docs/reports/2005/hiv.pdf]. Pretoria: South African Department of Health

3. Krug A, Pattinson RC, Power DJ: Why children die: an under-5 health care survey in Mafikeng region. S Afr Med J 2004, 94:202-206.

4. Jones N, Huebner R, Khoosal M, Crewe-Brown H, Klugman K: The impact of HIV on Streptococcus pneumoniae bacteraemia in a South African population. AIDS 1998, I2:2177-2184.

5. Madhi SA, Petersen K, Madhi A, Khoosal M, Klugman KP: Increased disease burden and antibiotic resistance of bacteria causing severe community-acquired lower respiratory tract infections in human immunodeficiency virus type I-infected children. Clin Infect Dis 2000, 31:170-176.

6. Meyers TM, Pettifor JM, Gray GE, Crewe-Brown H, Galpin JS: Pediatric admissions with human immunodeficiency virus infection at a regional hospital in Soweto, South Africa. J Trop Pediatr 2000, 46:224-230.

7. Kumar A, Teuber SS, Gershwin ME: Intravenous immunoglobulin: striving for appropriate use. Int Arch Allergy Immunol 2006 , 140:185-198.

8. Dalakas MC: Mechanisms of action of IVIg and therapeutic considerations in the treatment of acute and chronic demyelinating neuropathies. Neurology 2002, 59:SI3-S2I.

9. Lemieux R, Bazin R, Néron S: Therapeutic intravenous immunoglobulins. Mol Immunol 2005, 42:839-848.

10. NICHD: Intravenous immune globulin for the prevention of bacterial infections in children with symptomatic human immunodeficiency virus infection. The National Institute of Child Health and Human Developments Intravenous Immunoglobulin Study Group. N Engl J Med I99I, 325:73-80.

II. Spector SA, Gelber RD, McGrath N, Wara D, Barzilai A, Abrams E, Bryson Y], Dankner WM, Livingston RA, Connor EM: A controlled trial of intravenous immune globulin for the prevention of serious bacterial infections in children receiving zidovudine for advanced human immunodeficiency virus infection. Pedi- atric AIDS Clinical Trials Group. N Engl J Med 1994, 33I: II8I-II87.

12. El-Nawawy A, El-Kinany H, Hamdy El-Sayed M, Boshra N: Intravenous polyclonal immunoglobulin administration to sepsis syndrome patients: a prospective study in a pediatric intensive care unit. J Trop Pediatr 2005, 5 I :27 I-278.

13. Ohlsson A, Lacy JB: Intravenous immunoglobulin for suspected or subsequently proven infection in neonates. Cochrane Database Syst Rev 2004:CD00 I 239.

14. Jenson HB, Pollock BH: Meta-analyses of the effectiveness of intravenous immune globulin for prevention and treatment of neonatal sepsis. Pediatrics 1997, 99:E2.

15. Pildal J, Gøtzsche PC: Polyclonal immunoglobulin for treatment of bacterial sepsis: a systematic review. Clin Infect Dis 2004, 39:38-46.

16. Alejandria MM, Lansang MA, Dans LF, Mantaring JB: Intravenous immunoglobulin for treating sepsis and septic shock. Cochrane Database Syst Rev 2002:CD00 I090.

17. Stiehm ER: Shortage of IV immunoglobulin. Ann Allergy Asthma Immunol 2000, 85:424.

18. Milgrom H: Shortage of intravenous immunoglobulin. Ann Allergy Asthma Immunol 1998, 81:97-100.

19. Pollack MM, Patel KM, Ruttimann UE: PRISM III: an updated Pediatric Risk of Mortality score. Crit Care Med 1996, 24:743-752.

20. Shearer WT, Easley KA, Goldfarb J, Rosenblatt HM, Jenson HB, Kovacs A, Mclntosh K: Prospective 5-year study of peripheral blood CD4, CD8, and CDI9/CD20 lymphocytes and serum Igs in children born to HIV-I women. The P(2)C(2) HIV Study Group. J Allergy Clin Immunol 2000, 106:559-566.

21. Arango CA, Midani S, Alvarez A, Kubilis PS, Rathore MH: Usefulness of acute phase reactants in the diagnosis of acute infections in HIV-infected children. South Med ] 1999, 92:209-2 I3.

22. Waibale P, Bowlin SJ, Mortimer EA, Whalen C: The effect of human immunodeficiency virus- $I$ infection and stunting on measles immunoglobulin-G levels in children vaccinated against measles in Uganda. Int J Epidemiol 1999, 28:34I-346.

23. Gibb D, Giacomelli A, Masters J, Spoulou V, Ruga E, Griffiths H, Kroll $S$, Giaquinto $C$, Goldblatt $D$ : Persistence of antibody responses to Haemophilus influenzae type b polysaccharide conjugate vaccine in children with vertically acquired human immunodeficiency virus infection. Pediatr Infect Dis J 1996, I 5:1097-I IOI.

24. Lederman HM, Williams PL, Wu JW, Evans TG, Cohn SE, McCutchan JA, Koletar SL, Hafner R, Connick E, Valentine FT, McElrath MJ, Roberts NJ, Currier JS, AIDS Clinical Trials Group 889 Study Team: Incomplete immune reconstitution after initiation of highly active antiretroviral therapy in human immunodeficiency virus-infected patients with severe CD4+ cell depletion. Infect Dis 2003, 188: 1794-I803.

25. Koleba T, Ensom MH: Pharmacokinetics of intravenous immunoglobulin: a systematic review. Pharmacotherapy 2006, 26:8|3-827.

26. Mofenson LM, Moye J: Intravenous immune globulin for the prevention of infections in children with symptomatic human immunodeficiency virus infection. Pediatr Res 1993 , 33:S80-7. discussion S87-9

27. Blomberg B, Manji KP, Urassa WK, Tamim BS, Mwakagile DS, Jureen R, Msangi V, Tellevik MG, Holberg-Petersen M, Harthug S, Maselle SY, Langeland N: Antimicrobial resistance predicts death in Tanzanian children with bloodstream infections: a prospective cohort study. BMC Infect Dis 2007, 7:43.

28. Amadi B, Kelly P, Mwiya M, Mulwazi E, Sianongo S, Changwe F, Thomson M, Hachungula J, Watuka A, Walker-Smith J, Chintu C: Intestinal and systemic infection, HIV, and mortality in Zambian children with persistent diarrhea and malnutrition. J Pediatr Gastroenterol Nutr 200I, 32:550-554.

29. Kessler L, Daley H, Malenga G, Graham S: The impact of the human immunodeficiency virus type $I$ on the management of severe malnutrition in Malawi. Ann Trop Paediatr 2000, 20:50-56.

30. Heikens GT: How can we improve the care of severely malnourished children in Africa? PLoS Med 2007, 4:e45.

31. Deen JL, Funk M, Guevara VC, Saloojee H, Doe JY, Palmer A, Weber MW: Implementation of WHO guidelines on management of severe malnutrition in hospitals in Africa. Bull World Health Organ 2003, 81:237-243. 
32. Gardulf A: Immunoglobulin treatment for primary antibody deficiencies: advantages of the subcutaneous route. BioDrugs 2007, 21:105-116.

\section{Pre-publication history}

The pre-publication history for this paper can be accessed here:

http://www.biomedcentral.com/1471-2334/8/127/pre

pub

Publish with Bio Med Central and every scientist can read your work free of charge

"BioMed Central will be the most significant development for disseminating the results of biomedical research in our lifetime. " Sir Paul Nurse, Cancer Research UK

Your research papers will be:

- available free of charge to the entire biomedical community

- peer reviewed and published immediately upon acceptance

- cited in PubMed and archived on PubMed Central

- yours - you keep the copyright 